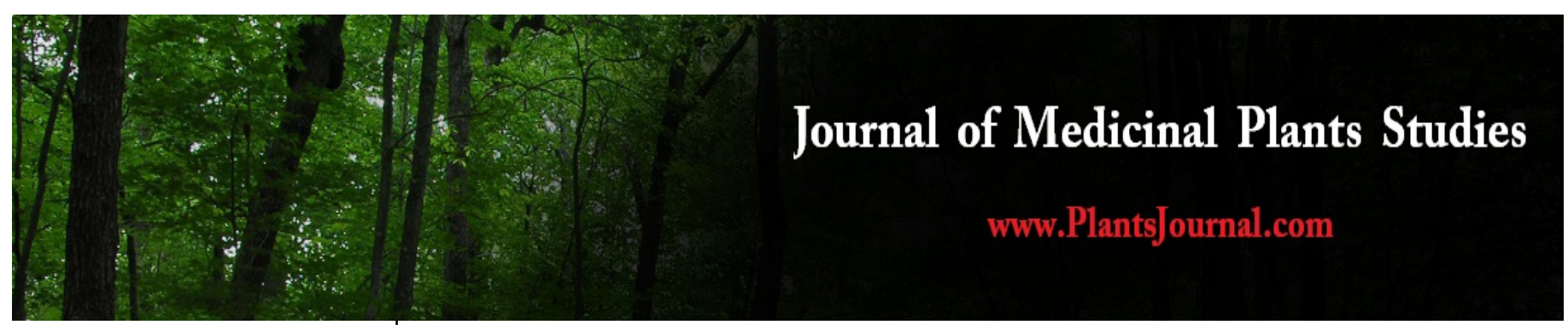

ISSN (E): 2320-3862 ISSN (P): 2394-0530

www.plantsjournal.com JMPS 2021; 9(4): 44-50

(C) 2021 JMPS

Received: 19-05-2020

Accepted: 21-06-2021

\section{Abdul Hadi}

Department of Botany,

University of Kashmir,

Hazratbal Srinagar, India

Seema Singh

Department of Botany,

University of Kashmir,

Hazratbal Srinagar, India

Corresponding Author:

Abdul Hadi

Department of Botany,

University of Kashmir,

Hazratbal Srinagar, India

\section{Ethno-botanical studies of some threatened medicinal plants and local perception of its population decline in Kargil, Ladakh UT}

\author{
Abdul Hadi and Seema Singh \\ DOI: https://doi.org/10.22271/plants.2021.v9.i4a.1311
}

\section{Abstract}

Kargil lies in the Trans-Himalayan region between $32^{\circ} 15^{`}-34^{\circ} 56^{`} \mathrm{~N}$ latitude and $75^{\circ} 35^{`}-76^{\circ} 57^{`} \mathrm{E}$ longitude covering a geographical area of $14,086 \mathrm{Km}^{2}$ has a vast diversity of aromatic and medicinal plants. The traditional uses of medicinal plants to cure various ailments in the Kargil district have been practiced from ancient times. The present study was conducted to validate the information on ethnobotanical studies of some threatened medicinal plants in the region and local people's perception of its population decline. Therefore extensive field survey was conducted in a different region of district Kargil of Ladakh UT from May 2015- September 2020 to observe the habitat, altitudinal ranges, flowering, traditional uses of threatened medicinal plants, and the factors which are operating for the depletion of these important taxa from their natural habitat. Interviews were conducted in Local languages from more than 1000 informants as the corresponding author is a native of the study area. A total of 22 threatened plant species (21Angiosperms, 1 gymnosperm) belongings to 17 genera, 14 families were traditionally used tribal peoples of the valley to cure various ailments. Most of the threatened taxa belong to the family Ranunculaceae followed by Asteraceae. From local people's perception, land-use change, illegal exploitation, and the selling of plant parts in the black market are the worst threat to the depletion of these species.

Significance Statement: The flora of Ladakh is crying for Conservation due to factors such as climate change, bio-piracy, smuggling, pre maturation harvesting pressure, poverty, unemployment, human interference which leads to their extinction in the near future. Therefore it's very pertinent to develop conservation strategies and adopt Sustainable utilization practices. For this mass awareness programs on sustainable utilization and conservation of these threatened medicinal plants need to be conducted by involving various stakes-holders, universities, colleges, schools, and other various government and nongovernmental organizations.

Keywords: Threatened plants, Kargil ethno-botany local perception conservation approaches.

\section{Introduction}

World Health Organization estimates that more than $75-80 \%$ population of the developing world depends on traditional medicine for primary healthcare ${ }^{[1,2]}$. WHO recorded more than 20,000 plant species globally used as medicine ${ }^{[3]}$. Medicinal plants also become a source of income for millions of people. Ethno-medicine has kept its fame in all regions of the developing world and its use is tremendously increasing in industrialized countries ${ }^{\text {[4] }}$. Ladakh is a newly formed Union Territory ( $5^{\text {th }}$ August 2019) separated from the state of Jammu \& Kashmir. It is considered as the "cold desert of India" due to its unique topographic, physiographic, and climatic conditions. It consists of two districts viz; Leh and Kargil which covers an area of more than $78,000 \mathrm{Km}^{2}$ which lies between $32^{\circ} 15^{`} 50-34^{\circ} 38 ` 11 \mathrm{~N}$ latitudes and $75^{\circ} 36^{`} 73-78^{\circ} 22^{`} 11 \mathrm{E}$ longitudes at an altitude of $2700-7560 \mathrm{~m}{ }^{[5-7]}$. Kargil lies in the Trans-Himalayan region between $32^{\circ} 15^{`}-34^{\circ} 56^{`} \mathrm{~N}$ latitude and $75^{\circ} 35^{`}-76^{\circ} 57^{`}$ E longitude covering a geographical area of $14,086 \mathrm{Km}^{2}$ possess huge diversity of temperate, alpine, and subalpine flora. Ethnobotanically Ladakh is explored by various authors such as [8-12]. However, they stick to the specific ethnic group. Kargil district is the ethnobotanical least explored region of Ladakh despite being vast socio-cultural and religious diversity. Besides this maximum floristic diversity in Ladakh are occurred in the Kargil district. Therefore in this present work, an attempt has been made with the assistance and collaboration of local peoples, herbalists, old aged peoples and herbal experts of various social and cultural tribes of Kargil 
district to substantiate a clear picture of traditional uses of some threatened medicinal plants and their perception and views on its population decline.

\section{Materials and Methods}

Study area: Kargil district is located between $32^{\circ} 15^{`}$ $34^{\circ} 56^{`} \mathrm{~N}$ latitude and $75^{\circ} 35^{`}$ - 76 ${ }^{\circ} 57^{`} \mathrm{E}$ longitude (Garmin etrex 10, 30) covering a geographical area of 14,086 sq. Km and shares its boundaries with Pakistan in the North, Leh district in the East, and Kashmir in the West. Extensive field surveys were conducted in a different region of the Kargil district of Ladakh UT from Zojila-pass to Zanskar. Which include the main valleys such as Drass, Hundurman, Batalik, Gargardo, Darchiks, Darkon, Chiktan, Shargole, Sapi, Soth, Kargil, TSG, Sankoo, Barsoo, Suru, Rangdum, and Zanskar from May 2015- September 2020. A field survey was conducted every year from the commencement of spring to the onset of autumn to gain maximum information about the distribution, habitat, flowering season, traditional uses and causes of population decline and to cross-check the information of informants of former visits. The accuracy and reliability of gathered information were confirmed by visiting and interviewing the informants five to six times. Videos, Photographs, and live specimens were shown to the informants such as herbalists, older peoples, plant experts, "Aabs, Amchis", to get relevant and authentic information on traditional uses (table 2). One question is asked strictly to every informant I. e., Did you noticed that the population of this particular species is declined day by day?, What is your perception about this? Data on elevation, latitude, longitude were recorded by GPS (Garmin etrex-30) specimen habit, habitat, and flowering season were recorded on the spot by keenly observing the specimen. All specimens were identified on the spot with the help of local floras, databases, standard literature, and with the help of regional taxonomic experts. Voucher specimens were deposited in Kashmir University KASH Herbarium, Department of Botany. The scientific name is checked from the database theplantlist.org.

\section{Result and Discussion}

Total 22 threatened plant species (21Angiosperms, 1 Gymnosperm) belongings to 17 genera, 14 families were traditionally used by tribal peoples of Kargil to cure various ailments (Table 2). Most of the threatened taxa belong to the family Ranunculaceae followed by Asteraceae (Figure2). Maximum threatened plants were herbs (91\%) followed by shrubs (9\%) (Figure1). Roots were the most used parts followed by leaves and whole plants (Figure 3). Most of the threatened species belong to alpine vegetation and luxuriantly grows in mesic to wet habitats (Table 2). Every species is used to cure various types of diseases, but most of them used to cure digestive and respiratory-related diseases (Table 2). According to the informant's perception, collection of rhizomes of Aconitum species and Dactylorhiza hatagirea, Arnebia euchroma, Bergenia sps, Picrorhiza kurrooa, and selling it in the black market is the main cause for the depletion of these important Texas. To obtain this useful plant parts the smuggler targeted the primary and middle school children's, and unemployed youths, to collect the roots and other useful parts of these plants. Useful plant parts were sold at different costs which are mentioned in (Table 1). Besides this, another factor that we observed from the informants is the pre-harvesting pressure and introduction of exotic fodder plants. Elderly and uneducated women have great knowledge of the traditional uses of medicinal plants in Ladakh. The knowledge of medicinal plants decreases significantly with the increases in the educational level of the informants. The new generation did not even know the vernacular name of plants growing is in their vicinity.

\section{Conclusions}

Kargil has a rich cultural and floristic diversity. Most of the area in kargil district falls under the transitional zone which possesses unique diversity. Most of the threatened plants were used to cure several diseases. The plants of Kargil were facing the tremendous threat of their extinction due to factors such as land use-change, climate change, bio-piracy, smuggling, pre maturation harvesting pressure, poverty, unemployment, human interference. These plants were in need of. Therefore it's very pertinent to develop conservation strategies and adopt Sustainable utilization practices. For this mass awareness programs on sustainable utilization and conservation of these threatened medicinal plants need to be conducted by involving various stalk-holders, universities, colleges, schools, and other various government and nongovernmental organizations.

\section{Acknowledgement}

The corresponding author acknowledges Council of Scientific and Industrial Research, New Delhi for fellowship to carry out the research work \& also grateful and pay a tribute to his grandfather Late Haji Ghulam Mohmmad Mustay Numsuru, for providing valuable information regarding flora, beside this, the authors are highly thankful to the indigenous peoples of Kargil from Drass to Zanskar valley; particularly the people of Suru-valley for cooperating and providing us invaluable information regarding the cited study.

Table 1: Price of some threatened plant parts in local market

\begin{tabular}{|c|c|c|}
\hline Scientific Name & Part sold & Local Price INR per kg \\
\hline Aconitum heterophyllum & Root tubers & Rs. 2500 \\
\hline Aconitum violaceum & Root tubers & Rs. 1000 \\
\hline Dactylorhiza hatagirea & Rhizome & Rs. $800-1000$ \\
\hline Hippophae rhamnoides & Fruits & Rs. 400 \\
\hline
\end{tabular}

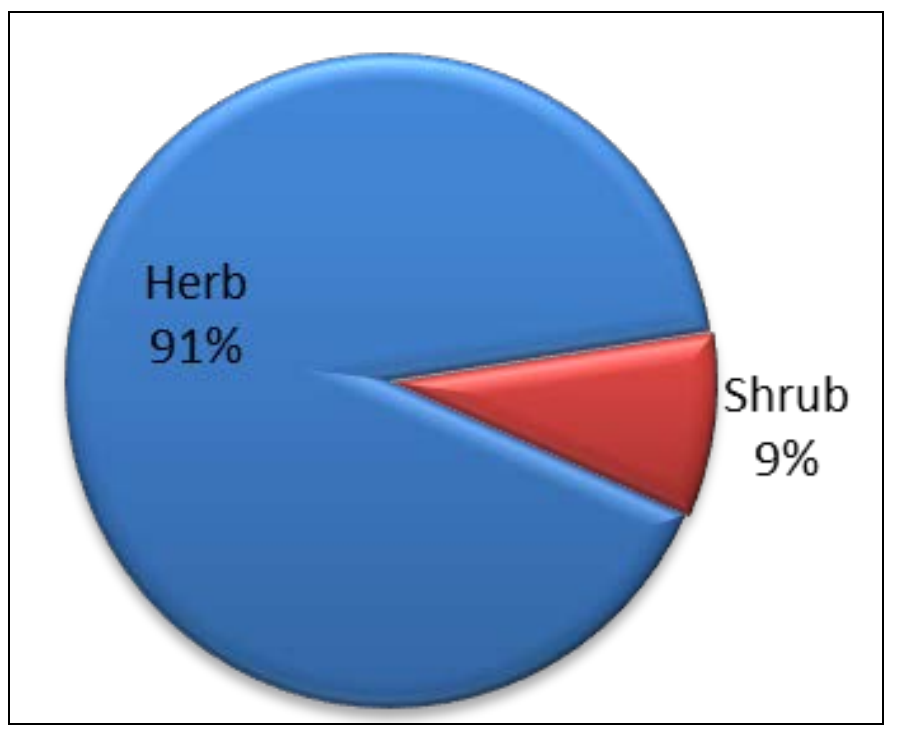

Fig 1: Pie chart showing habit of plant species in percentage 


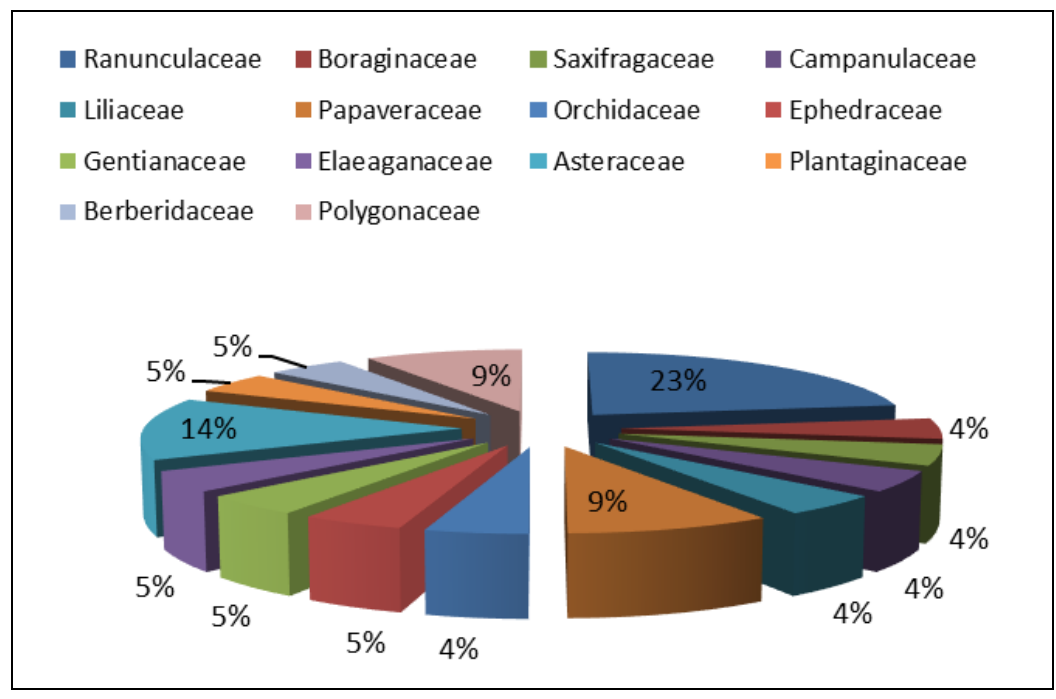

Fig 2: percentage contribution of threatened species

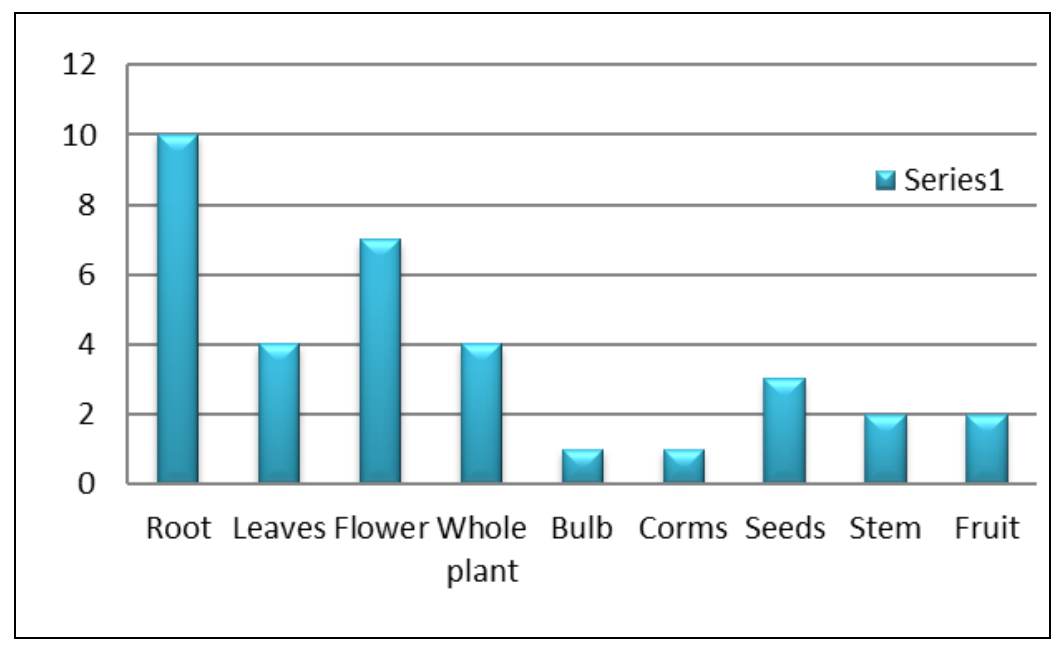

Fig 3: Plant parts used to cure ailments

Table 2: Ethno-medicinal uses of threatened medicinal plants in Kargil district of Ladakh Himalaya to cure various ailments by indigenous tribes.

\begin{tabular}{|c|c|c|c|c|c|c|c|c|c|}
\hline Scientific Name & Family & $\begin{array}{l}\text { Local } \\
\text { Name }\end{array}$ & Life form & \begin{tabular}{|c|}
$\begin{array}{c}\text { Altitude } \\
\text { (meter) } \\
\text { asl }\end{array}$ \\
\end{tabular} & Flowering & Habitat & $\begin{array}{l}\text { Part } \\
\text { used }\end{array}$ & $\begin{array}{c}\text { Medicinal uses/ and mode of } \\
\text { administration }\end{array}$ & $\begin{array}{l}\text { IUCN- } \\
\text { Status }\end{array}$ \\
\hline $\begin{array}{c}\text { Aconitum } \\
\text { heterophyllum } \\
\text { Wall. ex Royle }\end{array}$ & Ranunculaceae & $\begin{array}{c}\text { Karpo- } \\
\text { Bova/ } \\
\text { Bona-Karpo }\end{array}$ & $\mid \begin{array}{c}\text { Biennial } \\
\text { or } \\
\text { perennial } \\
\text { herb }\end{array}$ & $\begin{array}{l}3200- \\
3600\end{array}$ & $\begin{array}{c}\text { Mid-June - } \\
\text { July }\end{array}$ & $\begin{array}{c}\text { Moist damp soil, } \\
\text { semi-shaded places, } \\
\text { under the shade of } \\
\text { Salix species, moist } \\
\text { abandoned lands in } \\
\text { villages. }\end{array}$ & $\begin{array}{l}\text { Root } \\
\text { tuber }\end{array}$ & $\begin{array}{c}\text { Powder: Root tubers were ground into a } \\
\text { fine powder. A half teaspoon full of this } \\
\text { powder was taken orally along with one } \\
\text { glass of milk with an empty stomach } \\
\text { early in the morning to cure Toothache, } \\
\text { gastric disorders, and expulsion of } \\
\text { intestinal worms particularly Ascaris } \\
\text { lumbericoides. } \\
\begin{array}{c}\text { Decoction: Root decoction is useful to } \\
\text { relieve headache, intestinal complaint, } \\
\text { fever, diabetes, vomiting, and diarrhea. A } \\
\text { raw root tuber is kept between the teeth } \\
\text { to cure toothache. }\end{array} \\
\end{array}$ & Endangered \\
\hline $\begin{array}{c}\text { Aconitum } \\
\text { violaceum } \\
\text { Jacq.ex Stapf }\end{array}$ & Ranunculaceae & $\begin{array}{c}\text { Nagpo } \\
\text { Bova/ } \\
\text { Boma- } \\
\text { nagpo }\end{array}$ & $\begin{array}{l}\text { Biennial } \\
\text { herb }\end{array}$ & $\begin{array}{c}3200- \\
3800\end{array}$ & $\begin{array}{c}\text { Mid-June- } \\
\text { August }\end{array}$ & \begin{tabular}{|c|} 
Moist damp soil \\
along the bank of \\
irrigation canal, bank \\
of streams, springs, \\
semi-shaded damp \\
places. It is strictly \\
distributed along the \\
edges of continuous \\
springs and streams.
\end{tabular} & $\begin{array}{l}\text { Root } \\
\text { tubers }\end{array}$ & $\begin{array}{l}\text { Powder: } 10-15 \text { mg of root powdered is } \\
\text { used along with honey or milk to cure } \\
\text { cough, asthma, high fever, lung problem, } \\
\text { rheumatism, gastric problems. } \\
\text { Root paste: Paste of root powdered is } \\
\text { applied to cure boils and sciatic pains. } \\
\text { Decoction: Dried root powdered is used } \\
\text { to kill intestinal parasites. }\end{array}$ & Vulnerable \\
\hline $\begin{array}{l}\text { Aconitum } \\
\text { rotundifolium } \\
\text { Kar. \& Kir. }\end{array}$ & Ranunculaceae & $\begin{array}{c}\text { Ree- Bova/ } \\
\text { Ree-boma }\end{array}$ & $\begin{array}{l}\text { Biennial } \\
\text { herb }\end{array}$ & $\begin{array}{c}3800- \\
4440\end{array}$ & $\begin{array}{c}\text { Mid-July - } \\
\text { August }\end{array}$ & $\begin{array}{c}\text { Alpine grasslands, } \\
\text { bank of alpine } \\
\text { streams, stabilized } \\
\text { mesic to wet habitats. }\end{array}$ & $\begin{array}{l}\text { Root } \\
\text { tubers }\end{array}$ & $\begin{array}{l}\text { Powder: Half tea-spoon root powdered } \\
\text { is taken along with warm milk to cure } \\
\text { stomach trouble, intestinal worms, } \\
\text { abdominal pains, dizziness, indigestion, } \\
\text { blood purifier, and cough. }\end{array}$ & Vulnerable \\
\hline $\begin{array}{l}\text { Arnebia } \\
\text { euchroma }\end{array}$ & Boraginaceae & $\begin{array}{l}\text { Sgrons/ } \\
\text { sbrons/ }\end{array}$ & $\begin{array}{c}\text { Perennial } \\
\text { herbs }\end{array}$ & $\begin{array}{c}3550- \\
4840\end{array}$ & $\begin{array}{c}\text { Mid May- } \\
\text { August }\end{array}$ & $\begin{array}{c}\text { Stabilized sandy or } \\
\text { gravel slopes, screes, }\end{array}$ & $\begin{array}{l}\text { Root, } \\
\text { leaves }\end{array}$ & $\begin{array}{l}\text { Decoction: Dry root decoction is very } \\
\text { effective to cure Lungs problems, }\end{array}$ & Endangered \\
\hline
\end{tabular}




\begin{tabular}{|c|c|c|c|c|c|c|c|c|c|}
\hline $\begin{array}{l}\text { (Royle) I.M. } \\
\text { Johnst }\end{array}$ & & Demok & & & & $\begin{array}{l}\text { moistly found on } \\
\text { brownish sandy soil } \\
\text { on rocks, between } \\
\text { boulders, Arid to } \\
\text { semi-arid habitat. }\end{array}$ & & $\begin{array}{c}\text { pulmonary diseases, and purification of } \\
\text { blood, nose bleeding. } \\
\text { Extract: Roots were rinsed in mustard } \\
\text { oil for a week, and then it is applied to } \\
\text { overcome hair loss, strengthening hair, } \\
\text { and remove dandruff. Leaf powdered is } \\
\text { taken orally against dry cough. Root and } \\
\text { leaf paste is applied externally to relieve } \\
\text { back pains and healing of cuts and } \\
\text { wounds. }\end{array}$ & \\
\hline $\begin{array}{c}\text { Bergenia } \\
\text { stracheyi } \\
\text { (Hook.f. \& } \\
\text { Thomson) Eng. }\end{array}$ & Saxifragaceae & $\begin{array}{l}\text { Gatikpa/ } \\
\text { Shapur }\end{array}$ & $\begin{array}{c}\text { Perennial } \\
\text { herb }\end{array}$ & $\begin{array}{c}3680- \\
4300\end{array}$ & $\begin{array}{l}\text { June- } \\
\text { August }\end{array}$ & $\begin{array}{l}\text { Moist rocky slopes, } \\
\text { snow or stream-fed } \\
\text { rocks cervices, damp } \\
\text { sloppy steppes, semi- } \\
\text { arid to mesic habitat. }\end{array}$ & $\begin{array}{l}\text { Rhizome, } \\
\text { leaves, } \\
\text { flowers }\end{array}$ & \begin{tabular}{|} 
Powder: Roots are collected in the \\
month of August-September and shade \\
dried. A pinch of dried rhizome \\
powdered is then taken orally to dissolve \\
kidney stone, cure lung inflammation, \\
heartburn, menorrhagia, and urinary \\
related problems. Leaf paste is applied \\
externally on cuts and wounds. Root \\
paste is beneficial to relieve body pains. \\
Decoction: A cup of leaf and flower \\
decoction is effective against indigestion \\
and fever.
\end{tabular} & Vulnerable \\
\hline $\begin{array}{c}\text { Codonopsis } \\
\text { clematidea } \\
\text { Schrenk \& C.B. } \\
\text { Clarke }\end{array}$ & Campanulaceae & $\begin{array}{c}\text { Fak-fak/ } \\
\text { Mokhting }\end{array}$ & $\begin{array}{c}\text { Perennial } \\
\text { herbs }\end{array}$ & $\begin{array}{l}3150- \\
4100\end{array}$ & $\begin{array}{l}\text { July- } \\
\text { August }\end{array}$ & \begin{tabular}{|} 
Semi-arid to mesic \\
habitat, Mostly \\
present under the \\
shade of boulders, \\
stabilized moist \\
semi-shaded places \\
near inhabitants, \\
along the margins of \\
cultivated fields, \\
Near alpine stream \\
banks.
\end{tabular} & $\begin{array}{l}\text { Whole } \\
\text { plant }\end{array}$ & $\begin{array}{c}\text { Decoction: Root decoction is helpful to } \\
\text { cure stomach ulcer, GERD, Whole plant } \\
\text { part decoction is used to treat gout, liver } \\
\text { disorders, chest conjunction, nerves } \\
\text { disorder, rheumatism. } \\
\text { Paste: Root pastes are applied on cuts } \\
\text { and wound portions to stop bleeding and } \\
\text { healing of wounds. Flower and root are } \\
\text { consumed as raw by local peoples. }\end{array}$ & Rare \\
\hline $\begin{array}{l}\text { Colchicum } \\
\text { luteum Baker }\end{array}$ & Liliaceae & $\begin{array}{c}\text { Tukapa/ } \\
\text { Kapi-cherik }\end{array}$ & $\begin{array}{c}\text { Biennial } \\
\text { or } \\
\text { perennial } \\
\text { herb }\end{array}$ & $\begin{array}{l}3300- \\
3800\end{array}$ & $\begin{array}{c}\text { May-early } \\
\text { June }\end{array}$ & $\begin{array}{c}\text { Stabilized and un- } \\
\text { stabilized gravel or } \\
\text { sandy slopes when } \\
\text { melting of snow } \\
\text { brought abundant } \\
\text { moisture. } \\
\end{array}$ & $\begin{array}{l}\text { Bulb, } \\
\text { corms, } \\
\text { seeds, } \\
\text { flower }\end{array}$ & $\begin{array}{c}\text { Blub is directly consumed as it is } \\
\text { claimed to cure gout. Corms and seeds } \\
\text { are the rich sources of drug "colchicine" } \\
\text { which is used to treat inflammation, } \\
\text { cough, and fever. }\end{array}$ & Endangered \\
\hline $\begin{array}{c}\text { Corydalis } \\
\text { govaniana Wall }\end{array}$ & Papaveraceae & $\begin{array}{c}\text { Maqshang/ } \\
\text { Stongzil }\end{array}$ & $\begin{array}{c}\text { Perennial } \\
\text { herb }\end{array}$ & $\begin{array}{l}3780- \\
4550\end{array}$ & $\begin{array}{l}\text { July- } \\
\text { August }\end{array}$ & \begin{tabular}{|} 
Arid to mesic habitat, \\
Alpine meadows, \\
stony moist slopes \\
nearer to alpine \\
streams.
\end{tabular} & $\begin{array}{l}\text { Whole } \\
\text { plant } \\
\text { parts }\end{array}$ & \begin{tabular}{|} 
Decoction: plants were collected early \\
morning and sun-dried, then the \\
decoction of dries aerial shoots such as \\
leaves, flowers were given to a patient \\
suffering from back pain, joint pains, \\
cold, cough, abdominal pain, gastric \\
problems, constipation and also acts as a \\
blood purifier. Tribal peoples collected \\
them during the summer season and \\
stored them in dried shaded places for \\
usage in winter when getting necessary. \\
\end{tabular} & Vulnerable \\
\hline $\begin{array}{l}\text { Dactylorhiza } \\
\text { hatagirea } \\
\text { (D.Don) Soo }\end{array}$ & Orchidaceae & Angu-lakpa & $\begin{array}{c}\text { Perennial } \\
\text { herbs }\end{array}$ & $\begin{array}{l}3100- \\
3800\end{array}$ & $\begin{array}{c}\text { Mid-June- } \\
\text { July }\end{array}$ & $\begin{array}{l}\text { Wet muddy damp } \\
\text { soil, wet alpine } \\
\text { meadows, Banks of } \\
\text { streams and springs. }\end{array}$ & $\begin{array}{l}\text { Root } \\
\text { tubers }\end{array}$ & $\begin{array}{c}\text { Roots are directly consumed as it is } \\
\text { considered as rich sources of vitamins } \\
\text { and minerals. } \\
\text { Powder: Tubers are dried and ground } \\
\text { into powdered than it is given to } \\
\text { pregnant ladies, weak child, and old aged } \\
\text { peoples. It acts as a health tonic. Besides } \\
\text { this, its rhizome is claimed to be highly } \\
\text { beneficial to cure Kidney disorders, } \\
\text { fever, sedative, dysuria, diarrhea, cuts, } \\
\text { wounds, and roundworms. }\end{array}$ & $\begin{array}{c}\text { Critically } \\
\text { Endangered }\end{array}$ \\
\hline $\begin{array}{l}\text { Delphinium } \\
\text { brunonianum } \\
\text { Royle }\end{array}$ & Ranunculaceae & $\begin{array}{l}\text { Bila- } \\
\text { mindok }\end{array}$ & $\begin{array}{c}\text { Perennial } \\
\text { herbs }\end{array}$ & $\begin{array}{c}3900- \\
4900\end{array}$ & $\begin{array}{l}\text { July- } \\
\text { August }\end{array}$ & \begin{tabular}{|c|} 
Arid to mesic habitat, \\
screes, cervices of \\
big rocks, between \\
boulders on sandy \\
soil.
\end{tabular} & $\begin{array}{c}\text { Flower } \\
\text { and Seed }\end{array}$ & $\begin{array}{c}\text { Flower juice is used for curing colic } \\
\text { disorders. It is considered poisonous and } \\
\text { used as an insecticide and antiseptic. }\end{array}$ & Vulnerable \\
\hline $\begin{array}{c}\text { Delphinium } \\
\text { cashmerianum } \\
\text { Royle }\end{array}$ & Ranunculaceae & $\begin{array}{l}\text { Chagotpa/ } \\
\text { Bila- } \\
\text { mindok }\end{array}$ & $\begin{array}{c}\text { Perennial } \\
\text { herbs }\end{array}$ & $\begin{array}{l}3200- \\
4350\end{array}$ & $\begin{array}{l}\text { July- } \\
\text { August }\end{array}$ & \begin{tabular}{|} 
Mesic habitat, turf \\
soil along the banks \\
of irrigation canal, \\
moist stabilized \\
slopes on gravel soil.
\end{tabular} & & $\begin{array}{c}\text { Flower juice is used for curing colic } \\
\text { disorders. It is considered poisonous and } \\
\text { used as an insecticide and antiseptic. }\end{array}$ & Vulnerable \\
\hline $\begin{array}{c}\text { Ephedra } \\
\text { gerardiana } \\
\text { Wall. ex Stapf }\end{array}$ & Ephedraceae & $\begin{array}{l}\text { Chepaat/ } \\
\text { Tsepath }\end{array}$ & Shrub & $\begin{array}{l}3250- \\
3540\end{array}$ & \begin{tabular}{|c|} 
August- \\
September
\end{tabular} & $\begin{array}{l}\text { Hard stony stabilized } \\
\text { places, arid habitat, } \\
\text { screes at lower } \\
\text { elevation in Suru } \\
\text { valley and Rangdum }\end{array}$ & $\begin{array}{l}\text { Whole } \\
\text { plant }\end{array}$ & $\begin{array}{c}\text { Decoction: decoction of aerial shoots is } \\
\text { given to the patient suffering from } \\
\text { asthma, cough, rheumatism, pneumonia, } \\
\text { Ripened fruits are consumed as raw, as it } \\
\text { is claimed to be very beneficial for blood } \\
\text { purification, menstrual irregularities, } \\
\text { anti-cancerous and liver tonic. }\end{array}$ & Endangered \\
\hline $\begin{array}{c}\text { Gentiana algida } \\
\text { Pallas }\end{array}$ & Gentianaceae & Chukiting & $\begin{array}{c}\text { Perennial } \\
\text { herbs }\end{array}$ & $\begin{array}{l}3300- \\
3900\end{array}$ & $\begin{array}{l}\text { Mid-July- } \\
\text { September }\end{array}$ & $\begin{array}{c}\text { Wet alpine meadows, } \\
\text { grasslands, bank of } \\
\text { streams, damp snow }\end{array}$ & Flowers & \begin{tabular}{|c|}
$\begin{array}{c}\text { Decoction: Flower decoction was taken } \\
\text { orally before sleep to cure ailments like } \\
\text { esophagus infection inflammation of }\end{array}$ \\
\end{tabular} & Enda \\
\hline
\end{tabular}




\begin{tabular}{|c|c|c|c|c|c|c|c|c|c|}
\hline & & & & & & $\begin{array}{c}\text { bed pastures. Mosses } \\
\text { rich springs }\end{array}$ & & $\begin{array}{l}\text { stomach, lung, cough, sore throat, and } \\
\text { epidemic fevers. }\end{array}$ & \\
\hline $\begin{array}{l}\text { Hippophae } \\
\text { rhamnoides L }\end{array}$ & Elaeagnaceae & $\begin{array}{l}\text { Chak-ldum } \\
\text { /Cherma } \\
\text { /Sastaluloo }\end{array}$ & $\begin{array}{c}\text { Perennial } \\
\text { Shrubs }\end{array}$ & $\begin{array}{c}2850- \\
3800\end{array}$ & $\begin{array}{l}\text { Mid-June- } \\
\text { September }\end{array}$ & $\begin{array}{l}\text { Arid to mesic habitat, } \\
\text { along the bank river } \\
\text { deposited sandy and } \\
\text { gravel soil, stony } \\
\text { area, and abandoned } \\
\text { lands. }\end{array}$ & $\begin{array}{l}\text { Leaves, } \\
\text { flower, } \\
\text { fruits, } \\
\text { seeds }\end{array}$ & $\begin{array}{c}\text { Decoction: leaves and flower decoction } \\
\text { are used to cure ailments like lung } \\
\text { infection, cardiac disorders. Fruit and } \\
\text { seeds are consumed as it is claimed to be } \\
\text { rich sources of vitamins Such as Va, Vb, } \\
\text { Vc, Vk, carotenoids, and minerals. It is } \\
\text { believed to have anti-aging, anti- } \\
\text { cancerous properties, immune booster, } \\
\text { Fruit juice extract has been claimed to be } \\
\text { beneficial for diabetic patients, against } \\
\text { ulcers, joint pains, blood pressure, and } \\
\text { wounds. }\end{array}$ & Rare \\
\hline $\begin{array}{c}\text { Inula racemosa } \\
\text { Hook.f }\end{array}$ & Asteraceae & Manu & $\begin{array}{c}\text { Perennial } \\
\text { herbs }\end{array}$ & $\begin{array}{c}2800- \\
3500\end{array}$ & $\begin{array}{l}\text { July to } \\
\text { Late } \\
\text { August }\end{array}$ & $\begin{array}{c}\text { Mesic habitat, mostly } \\
\text { cultivated in Kargil } \\
\text { and Leh }\end{array}$ & $\begin{array}{l}\text { Root, } \\
\text { Leaves }\end{array}$ & $\begin{array}{c}\text { Powder: Dried root powdered were } \\
\text { taken along with warm water or milk to } \\
\text { cure stomach trouble, acidity, chest pain, } \\
\text { cold, fever. Root or leaf paste is applied } \\
\text { externally to heal the wound. }\end{array}$ & Endangered \\
\hline $\begin{array}{c}\text { Meconopsis } \\
\text { aculeata Royle }\end{array}$ & Papaveraceae & $\begin{array}{l}\text { Achay-na } \\
\text { numo- } \\
\text { mindok }\end{array}$ & $\begin{array}{c}\text { Perennial } \\
\text { herbs }\end{array}$ & $\begin{array}{c}4300- \\
4650\end{array}$ & $\begin{array}{c}\text { July- } \\
\text { September }\end{array}$ & $\begin{array}{c}\text { High Alpine screes, } \\
\text { between large rock } \\
\text { boulders, Semi-arid } \\
\text { to mesic habitat. } \\
\text { Moist snow beds } \\
\text { between }\end{array}$ & $\begin{array}{l}\text { Whole } \\
\text { plant } \\
\text { parts }\end{array}$ & $\begin{array}{c}\text { Extract: whole plant part extract is given } \\
\text { to the patient in small doses for a week } \\
\text { against gastric related discomforts. Leaf } \\
\text { paste is applied externally on swelling } \\
\text { portions of legs caused due to long } \\
\text { walks. }\end{array}$ & $\begin{array}{l}\text { Critically } \\
\text { Endangered }\end{array}$ \\
\hline $\begin{array}{c}\text { Picrorhiza } \\
\text { kurrooa Royle } \\
\text { ex Benth }\end{array}$ & Plantaginaceae & Kaor & $\begin{array}{c}\text { Perennial } \\
\text { herbs }\end{array}$ & $\begin{array}{c}3850- \\
4200\end{array}$ & $\begin{array}{l}\text { June- } \\
\text { August }\end{array}$ & $\begin{array}{c}\text { Alpine meadows, } \\
\text { moist alpine pastures }\end{array}$ & Root & $\begin{array}{c}\text { Rhizomes are collected washed and } \\
\text { shade dried completely and ground into } \\
\text { fine powdered which is then taken orally } \\
\text { along with one cup of warm water or } \\
\text { milk to kill roundworms and other } \\
\text { intestinal parasites, stomachs, and } \\
\text { intestinal infection. A decoction of root } \\
\text { powdered is considered to be beneficial } \\
\text { for diabetic and jaundice patients. It is } \\
\text { also used to cure burning urination and } \\
\text { kidney disorders. }\end{array}$ & Endangered \\
\hline $\begin{array}{l}\text { Podophyllum } \\
\text { hexandrum } \\
\text { Royle }\end{array}$ & Berberidaceae & $\begin{array}{l}\text { Dainmi- } \\
\text { koshu / } \\
\text { Tandik }\end{array}$ & $\begin{array}{c}\text { Perennial } \\
\text { herbs }\end{array}$ & $\begin{array}{c}3300- \\
4000\end{array}$ & May-June & $\begin{array}{c}\text { Mesic habitat, semi- } \\
\text { shaded damp soils, } \\
\text { under the shade of } \\
\text { Salix Species, }\end{array}$ & $\begin{array}{l}\text { Flower, } \\
\text { Fruit }\end{array}$ & $\begin{array}{c}\text { Dried as well as raw fruit was consumed } \\
\text { directly as it is believed to be helpful for } \\
\text { curing gynecological disease like } \\
\text { menstrual irregularity, improves lung and } \\
\text { blood circulation, helps in parturition, } \\
\text { skin diseases, anti-cancerous }\end{array}$ & Endangered \\
\hline $\begin{array}{l}\text { Rheum } \\
\text { spiciforme } \\
\text { Royle }\end{array}$ & Polygonaceae & Lhachu & $\begin{array}{c}\text { Perennial } \\
\text { herb }\end{array}$ & $\begin{array}{c}3500- \\
4800\end{array}$ & $\begin{array}{l}\text { July- } \\
\text { August }\end{array}$ & $\begin{array}{l}\text { Mesic alpine stony } \\
\text { slopes }\end{array}$ & $\begin{array}{l}\text { Root, } \\
\text { Stem }\end{array}$ & $\begin{array}{l}\text { The stem is consumed as raw as it has a } \\
\text { sour taste. It is used to treat swelling } \\
\text { wounds, rheumatism, fever, Internal } \\
\text { damages, chronic bronchitis, and piles. }\end{array}$ & Vulnerable \\
\hline $\begin{array}{l}\text { Rheum } \\
\text { webbianum } \\
\text { Royle }\end{array}$ & Polygonaceae & $\begin{array}{c}\text { Oma-khul/ } \\
\text { Lachoo }\end{array}$ & $\begin{array}{c}\text { Perennial } \\
\text { herb }\end{array}$ & $\begin{array}{c}3500- \\
4200\end{array}$ & $\begin{array}{l}\text { July- } \\
\text { August }\end{array}$ & $\begin{array}{l}\text { Alpine stony mesic } \\
\text { slopes, between } \\
\text { boulders. Screes, } \\
\text { alpine pastures }\end{array}$ & $\begin{array}{l}\text { Root, } \\
\text { Stem }\end{array}$ & \begin{tabular}{|c|} 
Root and stem were consumed as raw as \\
it was claimed to be rich sources of \\
vitamin C and ascorbic acid. A paste of \\
stem is applied to cure boils. Besides \\
this, it also acts as an appetizer, \\
astringent, purgative, health tonic. \\
\end{tabular} & Vulnerable \\
\hline $\begin{array}{c}\text { Saussurea } \\
\text { bracteata Decne }\end{array}$ & Asteraceae & $\begin{array}{l}\text { Spangsi- } \\
\text { tawo }\end{array}$ & $\begin{array}{c}\text { Perennial } \\
\text { herb }\end{array}$ & $\begin{array}{c}4200- \\
4800\end{array}$ & $\begin{array}{c}\text { Mid-July- } \\
\text { August }\end{array}$ & $\begin{array}{l}\text { Alpine meadows, } \\
\text { stony moist slopes }\end{array}$ & $\begin{array}{l}\text { Leaf, } \\
\text { flower } \\
\text { buds }\end{array}$ & $\begin{array}{c}\text { Paste: a paste of flower buds is applied } \\
\text { externally to cure boils, cuts, and } \\
\text { wounds, applied on the forehead to } \\
\text { relieve headache. }\end{array}$ & Vulnerable \\
\hline $\begin{array}{l}\text { Saussurea } \\
\text { costus (Falc.) } \\
\text { Lipsch. }\end{array}$ & Asteraceae & Rulta & $\begin{array}{c}\text { Perennial } \\
\text { herb }\end{array}$ & $\begin{array}{l}2700- \\
3600\end{array}$ & $\begin{array}{c}\text { July- } \\
\text { September }\end{array}$ & $\begin{array}{c}\text { Mostly cultivated in } \\
\text { Leh and Kargil }\end{array}$ & Root & $\begin{array}{c}\text { Roots were used to cure various ailments } \\
\text { such as cancer, diabetes, ulcer, kidney } \\
\text { disorders, central nerve problems, } \\
\text { Gastric troubles and dysentery }\end{array}$ & $\begin{array}{c}\text { Critically } \\
\text { Endangered }\end{array}$ \\
\hline
\end{tabular}
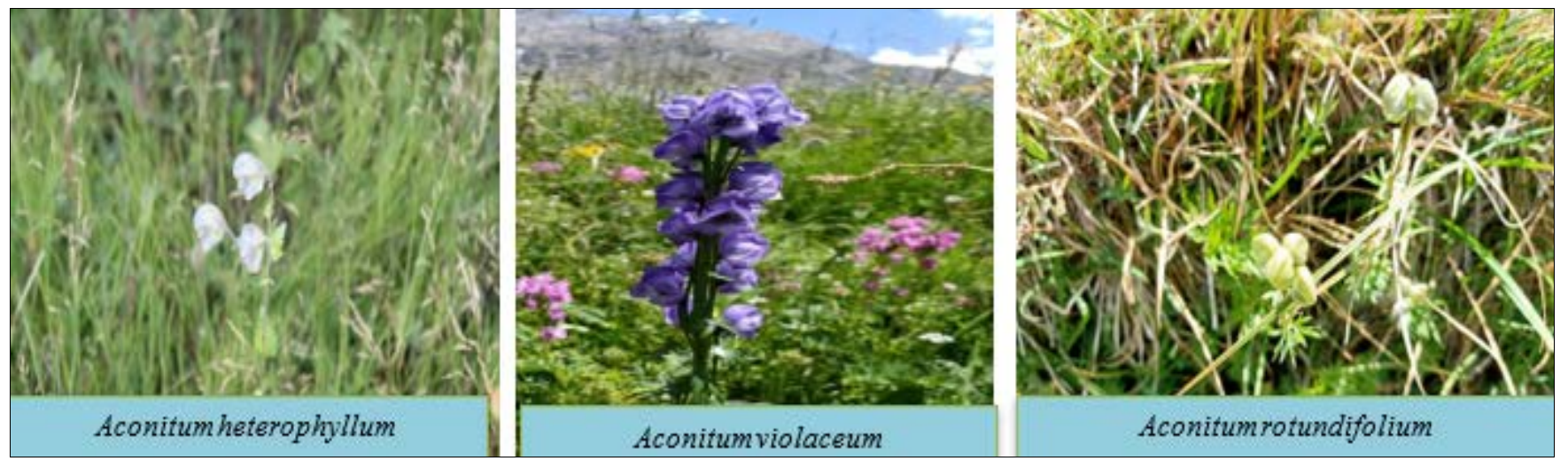


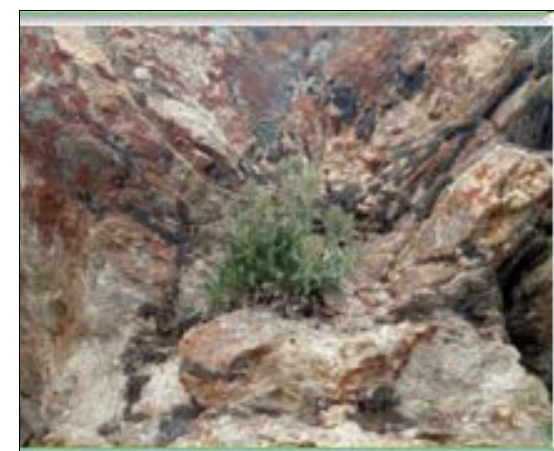

Arnahis auchroms

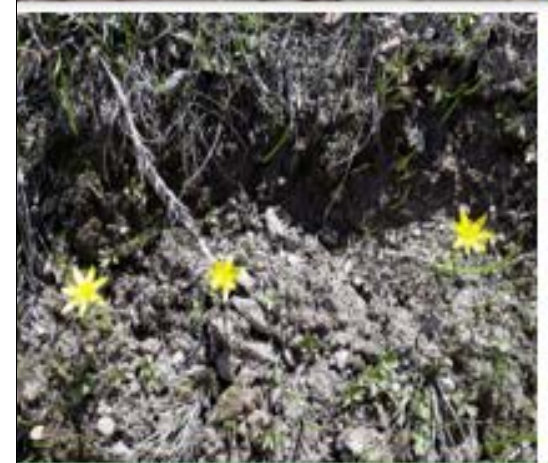

Colchicum luteum

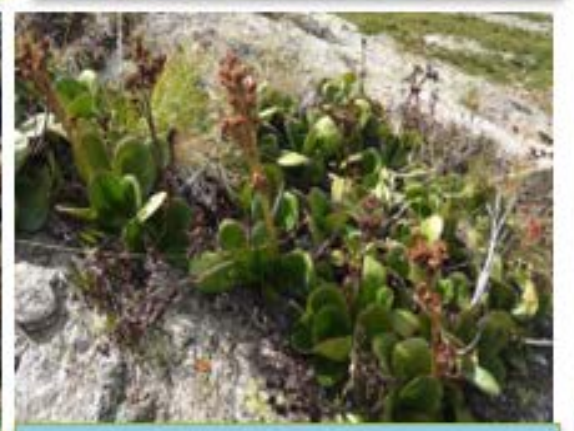

Bergenia stracheyi

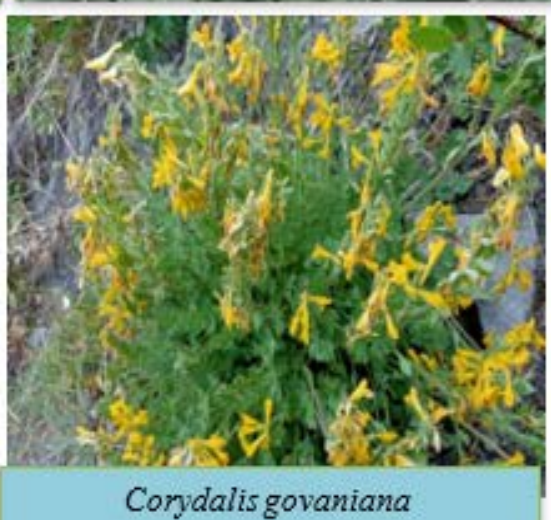

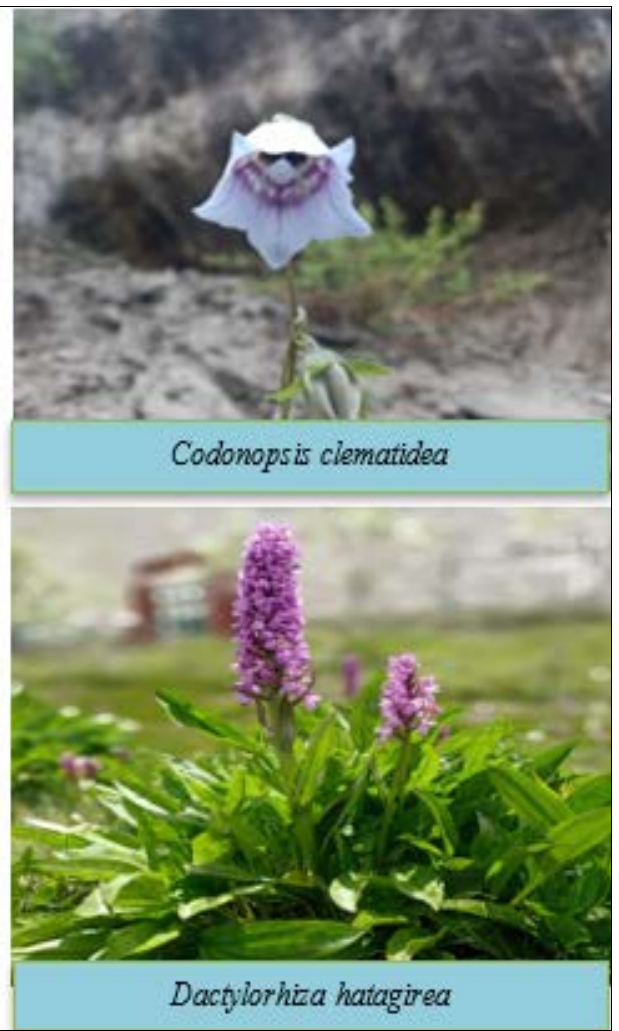

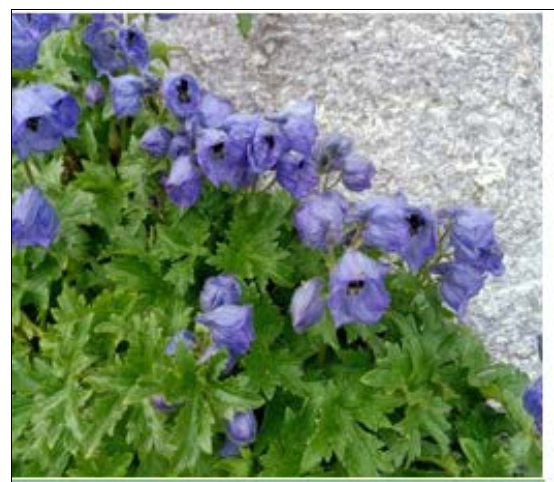

Delphinium brunonianum
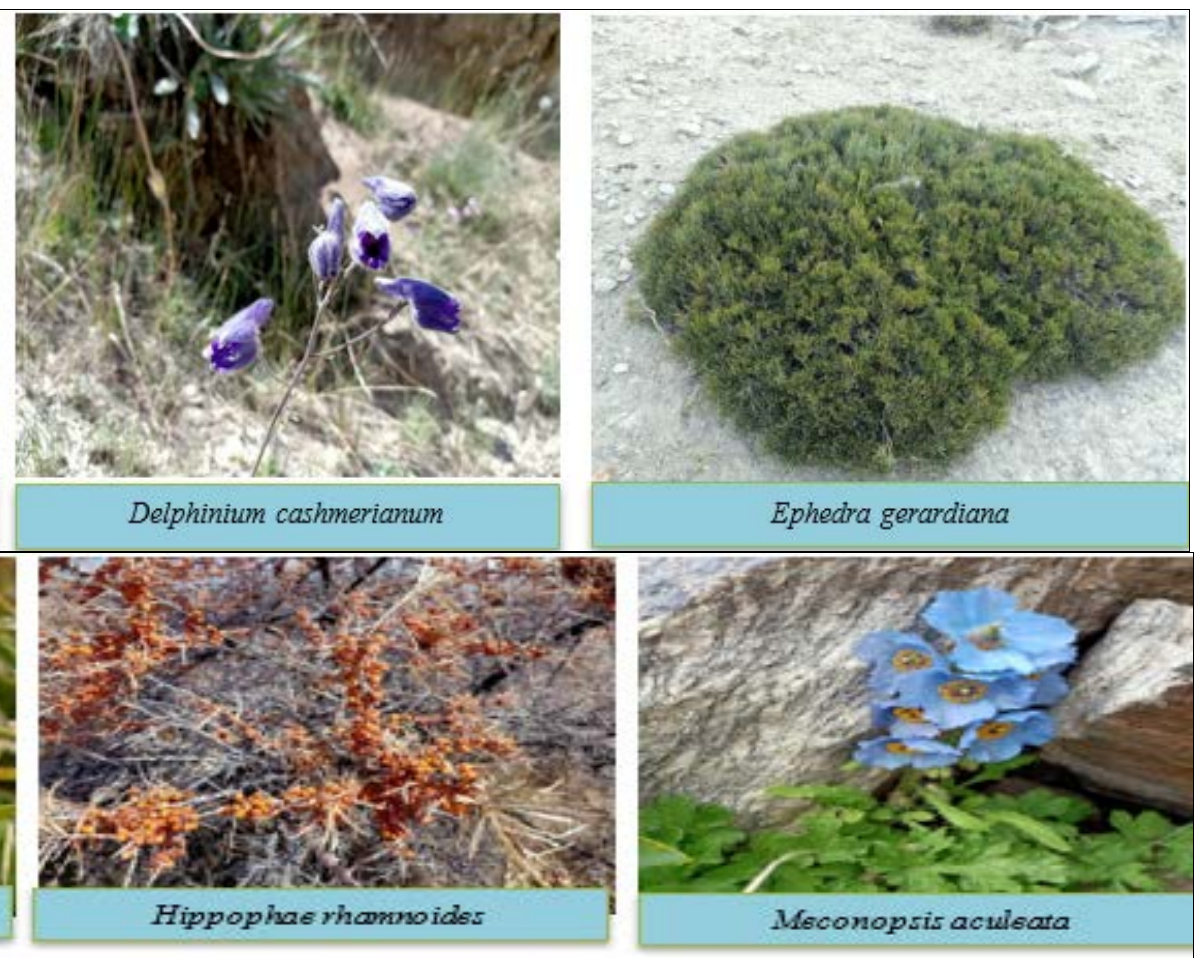

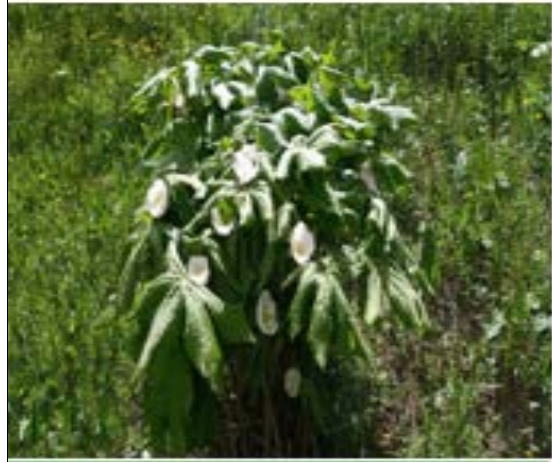

Podophyllwon hexandrum

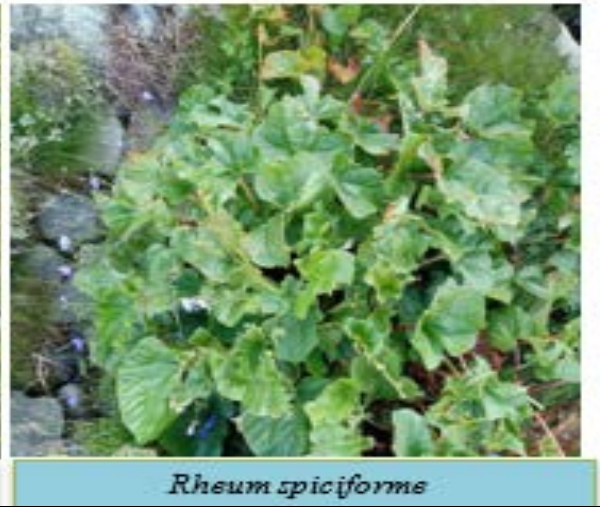




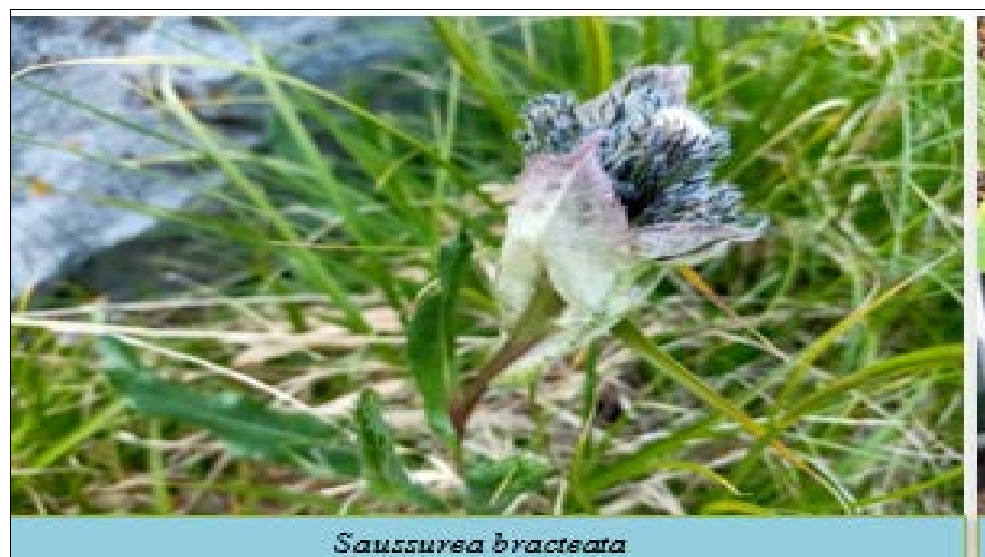

Saussurea bracteata

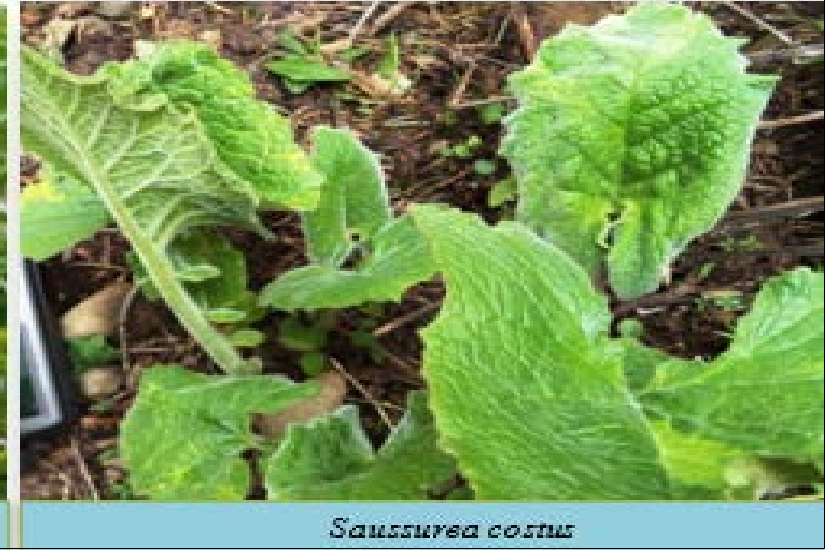

\section{References}

1. Famsworth NR, Akerele O, Bingel AS et al. Medicinal plants in therapy. Bulletin of the world health Organization 1985;63:965-981.

2. Singh JS. The biodiversity crisis. A multifaceted review. Curr Sci 2002;82(6):638.

3. Pandey MM, Rastogi S, Rawat AK. Indian herbal drug for general health care- An overview. The Internet Journal of Alternative Medicine 2008;6(1):1-10.

4. WHO World Health Organization Traditional Medicine Strategy 2002, 11.

5. Kala CP. Floral Diversity and Distribution in the High Altitude Cold Desert of Ladakh, India. J. Sustain. For. 2011;30:360-369. https://doi.org/10.1080/10549811.2011.534036

6. Stewart JL. I. Notes of a Botanical Tour in Ladak or Western Tibet. Trans. Bot. Soc. Edinburgh 1870;10:207239. https://doi.org/10.1080/03746607009468684

7. Hartmann H. A summarizing report on the phytosociological and floristical explorations (1976 1997) in Ladakh, India, 2009.

8. Angmo K, Adhikari BS, Rawat GS. Changing aspects of Traditional Healthcare System in Western Ladakh, India. J Ethnopharmacol 2012;143:621-630. https://doi.org/https://doi.org/10.1016/j.jep.2012.07.017

9. Ballabh B, Chaurasia OP. Herbal Formulations from Cold Desert Plants Used For Gynecological Disorders 2011;9:59-66.

10. Ballabh B, Chaurasia OP, Singh SB. Traditional medicinal plants of cold desert Ladakh-Used against kidney and urinary disorders 2008. https://doi.org/10.1016/j.jep.2008.04.022

11. Navchoo IA, Buth GM. Medicinal system of Ladakh, India. J Ethnopharmacol 1989;26:137-146. https://doi.org/https://doi.org/10.1016/03788741(89)90061-5

12. Namtak S, Sharma RC. Medicinal plant resources in Skuru watershed of Karakoram wildlife sanctuary and their uses in traditional medicines system of Ladakh India 2018. https://doi.org/10.15406/ijcam.2018.11.004 